\title{
Análisis de las reconsultas en un servicio de emergencia pediátrica
}

\author{
Mariana Mas*, Ana Casuriaga ${ }^{\dagger}$, Paola Cassanello $0^{\ddagger}$, Fernanda Salvo ${ }^{\ddagger}$, \\ Gustavo Giachetto§, Yelenna Ramírez, Susana Grunbaum
}

\section{Resumen}

Las reconsultas constituyen un indicador de calidad asistencial. Conocer sus características permite planificar estrategias para la mejora asistencial continua.

Objetivo: describir las características de las reconsultas en las primeras 72 horas en un servicio de emergencia pediátrica (SE) durante enero, abril, julio y octubre 2017.

Metodología: estudio descriptivo, retrospectivo, mediante revisión de historias clínicas electrónicas del SE de la Asociación Española. Variables: frecuencia, edad, sexo, momento, motivo de la reconsulta y evolución.

Resultados: en el período analizado ocurrieron 8.299 consultas y 476 reconsultas, 54 fueron excluidas por haber sido motivadas por patologías diferentes a la primera consulta. Se incluyeron 422, representando el 5,1\%. Media de edad: 4 años. Motivos de las reconsultas: persistencia de los síntomas $204(48 \%)$, evolución de la enfermedad $73(17 \%)$, control 59 (14\%), exámenes pendientes $51(12 \%)$, peoría $30(7 \%)$, diagnóstico diferente al planteado inicialmente $5(1 \%)$. Luego de la segunda consulta, el $88 \%$ fue dado de alta a domicilio y $11,5 \%$ hospitalizado. Ninguno de los niños ingresó a unidad de cuidado intensivo ni falleció.

Conclusiones: en este servicio la frecuencia de reconsultas es similar a la comunicada en otras series. Analizando los motivos de reconsulta, es necesario mejorar la gestión clínica desarrollando estrategias de comunicación con los padres y cuidadores y reforzando las consultas ambulatorias y la contrarreferencia al primer nivel de atención. Resulta importante reiterar el estudio luego de implementar planes de mejora de la gestión clínica.

Palabras clave: Indicadores de calidad de la atención de salud

Readmisión del paciente

Medicina de Urgencia Pediátrica

Reconsulta.

Key words: Quality indicators, Health care

Patient readmission

Pediatric Emergency Medicine

Reconsultation

\footnotetext{
* Prof. Adjunta del Departamento de Emergencia Pediátrica, Facultad de Medicina, Universidad de la República. Departamento de Atención Integral del Niño, Niña y Adolescente. Asociación Española.

† Asistente de Clínica Pediátrica, Facultad de Medicina, Universidad de la República. Departamento de Atención Integral del Niño, Niña y Adoles cente. Asociación Española.

¥ Departamento de Atención Integral del Niño, Niña y Adolescente. Asociación Española.

§ Prof. de Clínica Pediátrica, Facultad de Medicina, Universidad de la República.

Asociación Española, Primera en Salud, Montevideo, Uruguay.

Correspondencia: Dra. Ana Laura Casuriaga. Bulevar Artigas 1471, Montevideo, Uruguay. Correo electrónico: anitacasuriaga7@gmail.com

Los autores declaran no tener conflicto de interés.

Este trabajo fue aprobado por el Comité de Ética institucional.

Recibido: $7 / 1 / 19$

Aprobado: 22/4/19
} 


\section{Introducción}

En los últimos años la utilización de los servicios de emergencia hospitalarios (SE) de adultos y niños ha mostrado una tendencia creciente. Se han postulado múltiples factores como determinantes de esta tendencia. Entre ellos se destacan los cambios socioculturales y demográficos, modificaciones en los patrones de morbilidad, mayor accesibilidad a los SE, rapidez en la obtención de estudios complementarios e interconsultas con especialistas, y dificultades en el acceso al primer nivel de atención, entre otros ${ }^{(1-4)}$.

Sin embargo, al analizar los motivos de consulta, esta mayor demanda asistencial no siempre se encuentra justificada. Una proporción de los usuarios que consultan podría y debería resolver sus consultas mediante atención programada en servicios ambulatorios ${ }^{(1-4,5)}$.

La utilización inadecuada de los SE genera dificultades en la gestión clínica y en el funcionamiento de los servicios de salud en general y por lo tanto repercute en la calidad asistencial ${ }^{(2,6,7)}$.

Desde el año 2007, Uruguay atraviesa un proceso de reforma de su sistema de salud que promueve la universalización de la cobertura y el acceso, la equidad en el gasto y financiamiento con énfasis en la sostenibilidad del sistema y en la calidad asistencial. Uno de los ejes fundamentales de esta reforma es el cambio en el modelo de atención, reorientando los servicios y las acciones a un abordaje integral que contemple y priorice la promoción de salud, la prevención según riesgos específicos, el diagnóstico precoz y tratamiento oportuno, y la más adecuada recuperación de la salud. El cambio en el modelo de atención requiere la incorporación de la estrategia de atención primaria de la salud con mayor capacidad resolutiva del primer nivel de atención y la participación y satisfacción de los usuarios ${ }^{(8)}$. En este contexto resulta fundamental fortalecer la gestión clínica de los servicios desde una perspectiva de mejora continua de la calidad asistencial. Para ello es necesario incorporar un proceso sistemático y científico de evaluación mediante una serie de indicadores que den cuenta de la efectividad de las intervenciones, los procedimientos y los servicios en general. Es decir, la utilización de indicadores que permitan medir el grado en que los servicios logran cumplir con lo que se espera de ellos y los resultados de salud obtenidos en las circunstancias habituales ${ }^{(8,9)}$.

Los niños, niñas y adolescentes representan uno de los grupos priorizados por la reforma del sistema de salud en Uruguay. A pesar de ello, se dispone de escasa información en relación con la calidad de los servicios de atención pediátrica. Por lo tanto, resulta importante realizar relevamientos que den cuenta de la calidad de los
SE pediátricos, ya que representan uno de los servicios con mayor demanda y sobrecarga asistencial ${ }^{(9)}$.

Se han definido una serie de indicadores para evaluar la eficiencia y por ende la calidad de los procesos más frecuentemente utilizados en los SE: tasa de pacientes en cada nivel de triage, tiempo medio de primera asistencia, tiempo medio de permanencia en el SE, altas voluntarias, pacientes que se retiran sin ser asistidos, tasa de reclamos, grado de cumplimentación de la historia clínica, información a pacientes y familiares, proporción de ingresos, tasa de retornos o reconsultas y tasa de mortalidad en urgencias ${ }^{(11,12)}$.

La tasa de reconsultas en un período inferior a las 72 horas de la visita inicial se ha propuesto como un ejemplo de evaluación del proceso asistencial, dado que refleja la idoneidad del circuito de asistencia y está estrechamente relacionada con posibles problemas en la atención inicial $^{(1,11,12)}$.

Existen escasas comunicaciones internacionales que aborden el análisis de las reconsultas en un SE pediátrico y no hay disponibles hasta el momento datos locales acerca de este indicador. Es necesario obtener la información acerca de las reconsultas en el SE para poder detectar casos evitables, dificultades en la gestión de los pacientes y de ese modo planificar estrategias de mejora de la gestión clínica que impacten positivamente en la calidad asistencial $^{(1,11)}$.

La Asociación Española (AE) es un prestador del Sistema Nacional Integrado de Salud de Montevideo que cuenta con 188.000 usuarios de los cuales 25.395 son menores de 15 años. Para la atención pediátrica dispone de SE centralizado y un área de internación con 36 camas. El SE asiste a 30.000 consultas pediátricas anuales y el porcentaje anual de admisiones es de $8 \%$ (Departamento de Estadística, AE).

\section{Objetivo}

Describir las características de las reconsultas dentro de las 72 horas siguientes a la consulta inicial en el SE pediátrica de la AE durante los meses de enero, abril, julio y octubre de 2017.

\section{Metodología}

Se realizó un estudio descriptivo, retrospectivo, mediante revisión de las historias clínicas electrónicas de la AE. Se tomó una muestra por conveniencia de todas las consultas al SE pediátrica (menores de 15 años) durante un mes de cada estación del año (enero, abril, julio, octubre de 2017).

Se definió reconsulta cuando el paciente consultó nuevamente en el SE en un período inferior a las 72 horas siguientes a la visita inicial ${ }^{(1)}$. El cálculo de la tasa de reconsultas o readmisiones en 72 horas se realizó utili- 
zando la siguiente fórmula: Número de pacientes readmitidos en un intervalo de 72 horas/Número de pacientes asistidos en el SE en el período de estudio x 100. En el cálculo de la tasa de reconsultas e ingreso el numerador son los pacientes que reconsultan en el intervalo de 72 horas y son admitidos al hospital y el denominador son todos los pacientes que reconsultan en dicho intervalo. Las fórmulas utilizadas son las avaladas por las sociedades científicas internacionales, como la Sociedad Española de Urgencias de Pediatría (SEUP), entre otras.

En el análisis de los motivos de reconsulta se excluyeron las segundas visitas en el lapso referido por otra patología no vinculada con la consulta inicial.

Las variables analizadas fueron: frecuencia de las reconsultas, distribución por edad, sexo, patologías, motivo de reconsultas, destino final, causas de hospitalización.

Para el análisis del motivo de la reconsulta se consideraron las siguientes categorías: 1) Evolución de la enfermedad. Reconsulta debida a la evolución o agregado de síntomas y signos de la enfermedad que motivó la consulta inicial, sin cambios en la severidad. 2) Persistencia de los síntomas. Reconsulta debida a similares manifestaciones clínicas de la consulta inicial, sin agregado de nuevas manifestaciones, complicaciones o cambios en la severidad. 3) Peoría. Reconsulta previamente acordada para control evolutivo en el SE. 4) Exámenes o procedimientos pendientes. 5) Otro diagnóstico. Reconsulta por similares manifestaciones y se realiza otro diagnóstico diferente al planteado inicialmente.

Para la recolección de datos se utilizó una ficha electrónica precodificada elaborada por los autores.

Las variables continuas se describieron mediante media, mediana y rango, y las discretas con frecuencias relativas y absolutas. En la comparación de variables se consideró significativo $\mathrm{p}<0,05$. Para el análisis y procesamiento de datos se utilizó Epi Info 3.5.4.

El protocolo fue aprobado por el Comité de Ética de la institución. Se resguardó la confidencialidad de los datos en todas las etapas del estudio.

\section{Resultados}

Durante el período de estudio se registraron 8.299 consultas y correspondieron a reconsultas $5,7 \%(\mathrm{n}=476)$. La tasa de reconsulta e ingreso hospitalario fue de $11,5 \%$.

Se excluyeron 54 reconsultas que fueron motivadas por una patología diferente a la que motivó la consulta inicial. En los meses analizados reconsultaron por la misma patología 422 niños; las reconsultas representaron el 5,1\% de todas las consultas (IC95\% 3,0 - 7,2).

Las 422 reconsultas correspondieron a 383 niños: 352 niños consultaron en dos oportunidades, 23 niños en tres y 8 en cuatro.
Tabla 1. Análisis de las reconsultas en un servicio de emergencias pediátricas. Motivos de reconsulta en el SE.

\begin{tabular}{lcc}
\hline Motivo de reconsulta & $F A$ & $F R$ \\
\hline Persistencia de los síntomas & 204 & $48,5 \%$ \\
Evolución de la enfermedad & 73 & $17,5 \%$ \\
Controles & 59 & 14 \\
Procedimientos o exámenes pendientes & 51 & 12 \\
Peoría & 30 & 7 \\
$\begin{array}{l}\text { Diagnóstico diferente al planteado } \\
\text { inicialmente }\end{array}$ & 5 & 1 \\
\hline $\begin{array}{l}\text { SE: servicio de emergencia; FR: frecuencia relativa; FA: frecuen- } \\
\text { cia absoluta. }\end{array}$ & \\
\hline
\end{tabular}

La media de edad de los pacientes que reconsultaron fue 4 años y 10 meses, mediana 4 (rango 9 días-14 años). El $61 \%(n=260)$ era $\leq 5$ años y el $54 \%(n=230)$ de sexo masculino.

Las patologías por orden de frecuencia que motivaron las reconsultas fueron: infecciones respiratorias y crisis broncoobstructivas $37 \%(n=155)$, lesiones e injurias $14,5 \%(n=61)$, gastroenteritis $9 \%(n=40)$, fiebre sin foco clínico evidente $8 \%(n=34)$, infecciones de piel y tejidos blandos 8\% ( $\mathrm{n}=33)$, dolor abdominal $6 \%$ $(\mathrm{n}=27)$, infecciones urinarias $5 \%(\mathrm{n}=21)$, exantemas $4,5 \%(n=19)$, otros $8 \%(n=32)$.

Los motivos de las reconsultas fueron: persistencia de los síntomas 48,5\% ( $\mathrm{n}=204)$, evolución de la enfermedad $17,5 \%(n=73)$, controles $14 \%(n=59)$, procedimientos o exámenes pendientes $12 \%(\mathrm{n}=51)$, peoría $7 \%$ $(\mathrm{n}=30)$, otro diagnóstico $1 \%(\mathrm{n}=5)($ tabla 1$)$.

Las causas de las reconsultas por procedimientos o exámenes pendientes fueron: realización de curaciones (20/51), búsqueda de resultados de cultivos (16/51) y administración de medicación por vía parenteral (15/51).

La patología del grupo de 30 pacientes que consultaron por peoría fue: respiratoria 12 , infecciones de piel y tejidos blandos 9 , gastroenteritis $7 \mathrm{y}$ dolor abdominal 2 .

El destino de los 422 niños que reconsultaron fue alta a domicilio $88 \%(\mathrm{n}=371)$ y hospitalización en cuidados moderados $12 \%(\mathrm{n}=51)$.

En los pacientes que se hospitalizaron los motivos de las reconsultas fueron persistencia de los síntomas $(2 / 51)$, evolución de la enfermedad (27/51) y peoría (22/51). Los diagnósticos de los 51 pacientes hospitalizados fueron: bronquiolitis/crisis broncoobstructiva 22 , gastroenteritis 9 , exantema 5, dolor abdominal 5, cefalea 2 , problemas de salud mental 2 y otros, 4 . 
Entre los niños que reconsultaron no se registraron ingresos a unidad de cuidado intensivo ni fallecimientos durante el período de estudio.

Las patologías que motivaron más de dos consultas en el SE fueron: fiebre $\sin$ foco $(\mathrm{n}=9)$, lesiones $(\mathrm{n}=8)$, IU $(\mathrm{n}=3)$, OMA $(\mathrm{n}=3)$, gastroenteritis/vómitos $(\mathrm{n}=2)$, faringitis $(n=2)$, otros $(n=4)$. Los motivos de estas reconsultas reiteradas fueron procedimientos o exámenes pendientes $(n=15)$, control clínico $(n=10)$, persistencia de los síntomas $(n=4)$, evolución de la enfermedad $(n=1)$. Un paciente concurrió a control, se constató peoría e ingresó. No hubo otras hospitalizaciones en este grupo.

Al relacionar los motivos de reconsulta y las patologías, las infecciones respiratorias y crisis broncoobstructivas son la patología más frecuente de las reconsultas por persistencia de los síntomas 45\% $(\mathrm{n}=91)(\mathrm{p}<$ 0,05 ) y representan el $22 \%$ del total de las reconsultas. En segundo lugar las lesiones e injurias que reconsultan a control o realización de procedimientos representan el $9 \%(\mathrm{n}=37)$ del total de reconsultas. La patología respiratoria que reconsulta por evolución natural de la enfermedad ocupa el tercer lugar, $9 \%(\mathrm{n}=36)$.

\section{Discusión}

Los datos de este estudio permiten estimar que las reconsultas por la misma enfermedad en las primeras 72 horas en el SE pediátrica de este prestador integral de salud representan entre $3,0 \%$ y $7,2 \%$ de todas las consultas. La frecuencia de reconsultas observada es similar a la comunicada en otras series. Se destaca que $7 \%$ es el valor recomendado internacionalmente como estándar de calidad para este indicador, recientemente actualizado por la SEUP ${ }^{(6)}$.

$\mathrm{Al}$ analizar el porcentaje de hospitalizaciones se observó una diferencia estadísticamente significativa en la frecuencia de admisiones en los pacientes que reconsultaron $(11,5 \%$ vs $7 \%), p<0,05$. Este incremento de ingresos hospitalarios se reporta en todas las series y se podría atribuir a varias causas, vinculadas tanto a la patología que motiva el ingreso como a la mayor carga de angustia familiar que puede condicionar el proceso asistencial. Los pacientes que fueron hospitalizados reconsultaron mayoritariamente por evolución natural de la enfermedad o persistencia de los síntomas, sin peoría. Este hecho se vincula en muchos casos a la imposibilidad de asegurar un adecuado control o tratamiento ambulatorio. De todos modos, el tipo de diseño del estudio impide el adecuado análisis de estos aspectos ${ }^{(3)}$.

La patología respiratoria fue la más frecuente en los niños que reconsultaron. Este hallazgo coincide con la mayoría de las publicaciones que analizan las reconsultas en las primeras 72 horas. Bronquiolitis y crisis broncoobstructivas son los diagnósticos más frecuentes re- portados como causa de reconsulta. Es probable que la historia natural de estas patologías con evolución y persistencia de los síntomas por períodos prolongados pueda explicar estos hallazgos ${ }^{(7)}$.

$\mathrm{Al}$ considerar la distribución por edades de las reconsultas se observó que los pacientes $\leq 5$ años reconsultaron en primer lugar por infecciones respiratorias, mientras que los mayores de 5 años lo hicieron por dolor abdominal.

Analizados los motivos de reconsultas se observó que la principal causa de las reconsultas fue la persistencia de los síntomas, sin peoría de estos. Este hallazgo es un importante insumo para la planificación de estrategias de mejora de la calidad asistencial del SE, dado que si al momento de otorgar el alta a domicilio se le brinda a los padres o cuidadores del niño una adecuada información acerca de la posible evolución, signos de alarma y duración estimada de los síntomas, es probable que las reconsultas disminuyan.

Por otro lado, estos hallazgos son coincidentes con los de otras series, donde se observa que la mayoría de las reconsultas son motivadas por procesos banales o de poca gravedad que podrían ser asistidas y resueltas en consultas programadas en el primer nivel de atención con el médico de referencia ${ }^{(1,7)}$.

Un hecho cada vez más frecuente es la consulta con síntomas de reciente aparición. Esto hace más difícil establecer un diagnóstico nosológico y en ocasiones solo se realiza un diagnóstico sintomático, lo que conlleva mayor incertidumbre. La aparición de nuevos síntomas que constituyen la evolución natural de la enfermedad motiva un importante porcentaje de reconsultas en esta serie. El seguimiento por el pediatra de cabecera, fundamentalmente en estos procesos poco evolucionados, puede facilitar el diagnóstico, tratamiento precoz y una buena evolución de los pacientes ${ }^{(4)}$.

El tiempo de inicio de los síntomas en el momento de la primera consulta es un dato que no se registró.

La adecuada comunicación, con indicaciones al alta por escrito, con lenguaje simple y fácilmente comprensible por los padres o cuidadores podría contribuir a mejorar la adhesión y cumplimiento del tratamiento, disminuir la ansiedad familiar y evitar reconsultas. Un informe de alta adecuado es un indicador de calidad de los $\mathrm{SE}^{(1,4)}$

El pediatra de urgencias en ocasiones coordina con los padres un control clínico a las 24 o 48 horas de la consulta inicial. El SE no es el lugar indicado para citar a dichos pacientes a control, ya es probable que no requieran de los recursos diagnósticos y terapéuticos que se ofrecen en el SE y se encontrarán con un servicio sobrecargado y serán revalorados por un profesional diferente. Se propone agendar los controles clínicos de los ni- 
ños asistidos en el SE de ser posible con el pediatra tratante con una adecuada contrarreferencia o en una consulta planificada para tal fin independiente de la consulta espontánea de los SE. Las reconsultas por controles, al igual que aquellas motivadas por una patología distinta a la de la consulta inicial, frecuentemente son excluidas del indicador reconsultas, ya que no reflejan la calidad del proceso asistencial del SE. El principal motivo de las reconsultas reiteradas en el SE en esta serie fueron los controles y la realización de estudios o procedimientos pendientes. La instrumentación de una policlínica de procedimientos menores, como curación de heridas y la administración de medicamentos intramusculares, podría disminuir las reconsultas por estos motivos.

Otro factor que puede contribuir al crecimiento de las consultas y reconsultas en los SE es la actividad laboral de ambos padres. Esto se vincula con la sobrecarga de los SE especialmente en el horario vespertino ${ }^{(7)}$. La ampliación de los horarios de consultorios de atención primaria podría facilitar los controles por patologías agudas fuera del SE. En este estudio no se incluyó el análisis del horario de las reconsultas.

Es necesario profundizar en el análisis del proceso asistencial de los niños que reconsultaron por peoría y de aquellos en los que se realizó otro diagnóstico. La auditoría clínica, junto con el equipo del SE, representa una herramienta esencial para identificar fortalezas y debilidades en el proceso asistencial. Solo de esta forma se puede contribuir a la mejora continua de las prácticas y a evitar los errores médicos y mejorar la seguridad del paciente.

Constituyen limitantes de este estudio la falta de aleatorización en la selección de la muestra que influye en la representatividad y generalización de los resultados, así como el diseño retrospectivo que limita la cantidad de información para describir en forma más precisa otras características de las reconsultas. A diferencia de otras series, en este estudio no fueron analizadas las interconsultas, procedimientos, estudios complementarios y tratamientos realizados.

Independientemente de estas limitaciones, los resultados permiten una aproximación a la magnitud y característica del problema para poder implementar algunas estrategias de mejora.

Futuros estudios serán necesarios para profundizar en el análisis de este indicador y otros que permitan evaluar y mejorar la calidad asistencial del SE.

\section{Conclusiones}

En este SE pediátrica la frecuencia estimada de reconsultas es similar a la comunicada a nivel internacional y se encuentra dentro de los estándares de calidad. La per- sistencia de los síntomas y evolución esperada de la enfermedad fueron los principales motivos. Frente a estos hallazgos resulta necesario revisar las prácticas asistenciales, fortalecer la comunicación a padres o cuidadores y mejorar la contrarreferencia al primer nivel de atención. Resulta fundamental implementar planes de mejora y evaluar su impacto en futuros estudios.

\section{Abstract}

Reconsultations are an indicator of the quality of care. Learning about their characteristics allows planning strategies for continuous quality of care improvement.

Objective: to describe the characteristics of reconsultations within the first 72 hours in a Pediatric Emergency (PE) service during January, April, July and October of 2017.

Methods: descriptive, retrospective study through the review of electronic medical records of the PE service of a private health care provider in Montevideo. Variables: frequency, age, sex, time, reason for consultation and evolution.

Results: During the time analyzed, there were 8299 visits and 476 reconsultations. 54 reconsultations were excluded because they were due to conditions other than the one in the initial visit. 422 were included in the study, representing 5.1\%. Median age was 4 years. Reasons for reconsultations: persistence of symptoms 204 (48\%), disease evolution 73 (17\%), control 59 (14\%), tests pending $51(12 \%)$, worsening $30(7 \%)$, a different diagnosis $5(14 \%)$. After the second visit $88 \%$ were discharged to their homes and $11.5 \%$ were admitted. No child was referred to the intensive care unit, and none of them died.

Conclusions: The rate of reconsultations in this service is similar to that reported in other series. Upon analyzing the reason for reconsultations, it is necessary to improve clinical management, developing communication strategies with parents and carers and strengthening outpatient visits and counter-referral to the first level of care. It is important to repeat the study after the implementation of plans to improve clinical management.

\section{Resumo}

As reconsultas constituem um indicador da qualidade do atendimento. Conhecer suas características permite planejar estratégias para melhoria contínua do cuidado.

Objetivo: descrever as características de reconsultas dentro das 72 horas em um Serviço de Emergência Pediátrica (EP) em Janeiro, Abril, Julho e Outubro de 2017.

Metodologia: estudo descritivo, retrospectivo, através da revisão de registros clínicos da EP de um prove- 
dor de saúde privada em Montevidéu. Variáveis: frequência, idade, sexo, momento, motivo da reconsulta e evolução.

Resultados: no período analisado houve 8299 consultas e 476 reconsultas, sendo 54 excluídas por motivarem-se por patologias diferentes da primeira consulta. 422 foram incluídos, representando 5,1\%. Mediana de idade 4 anos. Razões para reconsultas: sintomas persistentes $204(48 \%)$, progressão da doença 73 (17\%), controle $59(14 \%)$, estudos pendentes $51(12 \%)$, peoria 30 (7\%), um outro diagnóstico 5 (1\%). Após a segunda consulta, $88 \%$ receberam alta hospitalar e $11,5 \%$ foram hospitalizados. Nenhuma das crianças entrou na unidade de terapia intensiva ou morreu.

Conclusões: neste serviço, a frequência de reconsultas é semelhante à relatada em outras séries. Analisando as razões da reconsulta, é necessário melhorar o manejo clínico, desenvolvendo estratégias de comunicação com pais e cuidadores e reforçando as consultas ambulatoriais e contrarreferência para o primeiro nível de atenção. É importante reiterar o estudo após a implementação de planos para melhorar o manejo clínico.

\section{Bibliografía}

1. Reynoso N, Fattore M, Hourbeigt M, González M, García Domínguez R. Análisis de los pacientes que reconsultaron a un Servicio de Emergencias Pediátricas de un Hospital Materno Infantil como indicador de calidad asistencial. Med Infantil 2015; 22(3):219-25.

2. Oterino D, Peiró S, Calvo R, Sutil P, Fernández O, Pérez G, et al. Utilización inadecuada de un servicio de urgencias hospitalario. Una evaluación con criterios explícitos. Gac Sanit 1999; 13(5):361-70.

3. Guardia M, Costa J, Pizá A, García J, Pou J, Luaces C. Análisis de los pacientes readmitidos con ingreso como indicador de calidad asistencial de un servicio de urgencias pediátricas. Emergencias 2013; 15:351-6.
4. Mintegui S, Benito J, Vázquez MA, Ortiz A, Capapé A, Fernández A. Los niños que repiten consulta en urgencias de pediatría. An Esp Pediatr 2000; 52:542-7.

5. Ali AB, Place R, Howell J, Malubay SM. Early pediatric emergency department return visits: a prospective patient-centric assesment. Clin Pediatr (Phila) 2012; 51:651-8.

6. Benito F, Fernández M, González S, Luaces C, Velazco R, González A, coord. Indicadores de calidad SEUP (Revisión 2018). Madrid: Sociedad Española de Urgencias de Pediatría, 2018.

7. Rivas García A, Manrique Martín G, Butragueño Laiseca L, Mesa García S, Campos Segura A, Fernández Iglesia V, et al. Hiperfrecuentadores en urgencias. ¿Quiénes son? ¿Por qué consultan? An Pediatr (Barc) 2017; 86:67-75.

8. Uruguay. Ministerio de Salud Pública, González T, Olesker D, comps. La construcción del Sistema Nacional Integrado de Salud 2005-2009. Montevideo: MSP, 2010. Disponible en: https://psico.edu.uy/sites/default/files/cursos/nas_la_ construccion.pdf [Consulta: 14 oct. 2018].

9. Mokate K. Eficacia, eficiencia, equidad y sostenibilidad: ¿qué queremos decir? Washington D.C.: BID, 1999. Disponible en: https://www.cepal.org/ilpes/noticias/paginas/9/ 37779/gover_2006_03_eficacia_eficiencia.pdf [Consulta: 7 nov. 2018].

10. Jiménez-Puente A, Del Río-Mata J, Arjona-Huertas JL, Mora-Ordóñez B, Nieto-de Haro L, Lara-Blanquer A, et al. Causas de los retornos durante las 72 horas siguientes al alta de urgencias. Emergencias 2015; 27:287-93.

11. Grupo de Trabajo SEMES-Insalud. Calidad en los servicios de urgencias: indicadores de calidad. Emergencias 2001; 13:60-5.

12. Alcaraz Martínez J, Blanco Linares M, González Soler M, López Picazo T, Lorenzo Zapata M, Martínez Bienvenido E. Eventos adversos en los servicios de urgencias: revisión de reconsultas como fuente de datos. Rev Calid Asist 2010; 25(3):129-35. 